\title{
Normalized Laplace Operators for Hypergraphs with Real Coefficients
}

\author{
Jürgen Jost ${ }^{1,2}$ and Raffaella Mulas ${ }^{* 1,3,4}$ \\ ${ }^{1}$ Max Planck Institute for Mathematics in the Sciences, Leipzig, \\ Germany \\ ${ }^{2}$ Santa Fe Institute for the Sciences of Complexity, New Mexico, USA \\ ${ }^{3}$ The Alan Turing Institute, London, UK \\ ${ }^{4}$ University of Southampton, Southampton, UK
}

\begin{abstract}
Chemical hypergraphs and their associated normalized Laplace operators are generalized and studied in the case where each vertex-hyperedge incidence has a real coefficient. We systematically study the effect of symmetries of a hypergraph on the spectrum of the Laplacian.
\end{abstract}

Keywords: Chemical hypergraphs, Laplace operator, Spectral theory

\section{Introduction}

The essential structure of many empirical systems consists in pairwise interactions between their elements, and such network structures are therefore mathematically as (possibly weighted and/or directed) graphs. The theory of such networks is well developed, and it has proven successful in many applications, see for instance [1. Many other systems, however, naturally support higher order interactions. In particular, many social networks involve interactions between groups of varying size. Scientific collaboration networks, where data are readily available from preprint repositories, are a standard example. Also, chemical reactions typically involve several educts, several products and perhaps some catalysts. Such structures can no longer be modelled by graphs, but should rather be considered as hypergraphs. In a hypergraph, a hyperedge can connect several vertices, and if we refine the structure a bit, we can in addition distinguish two classes of vertices, like the educts and products in a chemical reaction. While for a long time, hypergraphs have received less attention than ordinary graphs, this has recently changed, and the mathematical study of hypergraphs and its application to empirical systems has become very active, see for instance [2]. As for the qualitative analysis of graphs

*Email address: r.mulas@soton.ac.uk 
(see for instance [3]), the investigation of the spectrum of a Laplace operator can reveal important structural properties. In this contribution, we want to refine those tools, by looking at hypergraphs with real (and possibly negative) coefficients and studying the effects of symmetries on the spectrum. That is, we shall ask to what extent we can get indications of the presence (or absence) of symmetries by looking at the spectrum of a hypergraph.

Let us now describe the setting of this paper in more concrete terms. In 4 ] we introduced chemical hypergraphs with the aim of modelling chemical reaction networks. While classical hypergraphs are given by vertices and sets of vertices called hyperedges, chemical hypergraphs have the additional structure that each vertex has either a plus sign, or a minus sign, or both, for each hyperedge in which it is contained. The idea is that each hyperedge represents a chemical reaction involving the elements that it contains as vertices; plus signs are assigned to the educts or inputs of the reaction, minus signs are given to its products or outputs, and both signs are given to the catalysts participating in the reaction. We also introduced two normalized Laplace operators for chemical hypergraphs, the vertex Laplacian $L$ and the hyperedge Laplacian $L^{H}$, as a generalization of the classical theory introduced by F. Chung [5] for graphs. The spectra of these two operators, that only differ from each other by the multiplicity of the eigenvalue 0 , have been largely studied in [6-13].

Similarly, N. Reff and L. Rusnak [14] introduced oriented hypergraphs, i.e., hypergraphs in which each vertex in a hyperedge has either a plus or a minus sign, as well as a multiplicity: a natural number assigned to the given vertex-hyperedge incidence. They also developed a spectral theory of oriented hypergraphs that focuses on the spectra of the (unnormalized) Laplace operator $\Delta$ and the adjacency matrix $A$. This theory has also been widely studied (see for instance [14-27]) and, as pointed out in [8, Remark 2.17], for chemical hypergraphs without catalysts which are regular, meaning that all vertices have the same degree, the spectra of $L, \Delta$ and $A$ are all equivalent, up to a multiplicative or additive constant. For non-regular chemical hypergraphs, however, the spectra of these operators are in general not equivalent, implying that they encode different properties of the hypergraph to which they are associated.

Here we propose a further generalization of chemical hypergraphs and their associated operators, and we focus on the study of the normalized Laplacians. In particular, we consider hypergraphs in which each vertex-hyperedge incidence has a real coefficient.

Structure of the paper. In Section 2 we establish the basic definitions for hypergraphs with real coefficients and their associated operators, as a natural generalization of the constructions in [4]. In Section 3 we investigate the first properties of the generalized normalized Laplacians and in Section 4 we discuss the multiplicity of the eigenvalue 0. In Section 5 we apply the min-max principle in order to characterize all the eigenvalues in terms of the Rayleigh Quotient, and in Section 6 we investigate the largest eigenvalue. Finally, in Section 7 we give some general bounds on all eigenvalues; in Section 8 and Section 9 we study the effect of symmetries on the spectra of the generalized normalized Laplacians. 


\section{Basic definitions}

Definition 2.1. A hypergraph with real coefficients is a triple $\Gamma=(V, H, \mathcal{C})$ such that:

- $V=\left\{v_{1}, \ldots, v_{N}\right\}$ is a finite set of nodes or vertices;

- $H=\left\{h_{1}, \ldots, h_{M}\right\}$ is a multiset of elements $h_{j} \in \mathcal{P}(V)$ called hyperedges;

- $\mathcal{C}=\left\{C_{v, h}: v \in V\right.$ and $\left.h \in H\right\}$ is a set of coefficients $C_{v, h} \in \mathbb{R}$ and it's such that

$$
C_{v, h}=0 \Longleftrightarrow v \notin h \text {. }
$$

Remark 2.2. The chemical hypergraphs in [4 have coefficients $C_{v, h} \in\{-1,0,+1\}$ without the assumption (1), while the oriented hypergraphs defined by Reff and Rusnak in [14] can be seen as hypergraphs with coefficients $C_{v, h} \in \mathbb{Z}$. Moreover, a signed graph is a hypergraph with real coefficients such that:

- $H$ is a set (that is, $j \neq k$ implies $h_{j} \neq h_{k}$ );

- Each hyperedge contains exactly two vertices;

- $C_{v, h} \in\{+1,-1\}$ for all $h \in H$ and $v \in h$.

A simple graph is a signed graph such that, for each hyperedge $h$, there exists a unique $v \in h$ such that $C_{v, h}=1$ and there exists a unique $w \in h$ such that $C_{w, h}=-1$.

Remark 2.3. In the case when, for all $v$ and $h, C_{v, h} \geq 0$ and $\sum_{h \in H} C_{v, h}=1$, we can see each coefficient $C_{v, h}$ as the probability of the vertex $v$ to belong to the hyperedge $h$. In the case of a hypergraph with integer coefficients, we can see each vertex as a chemical element, each hyperedge as a chemical reaction and each coefficient $C_{v, h}$ as the chemical stoichiometric coefficient of the element $v$ in the reaction $h$.

Definition 2.4. We say that $\Gamma=(V, H, \mathcal{C})$ is connected if, for every pair of vertices $v, w \in V$, there exists a path that connects $v$ and $w$, i.e. there exist $v_{1}, \ldots, v_{m} \in V$ and $h_{1}, \ldots, h_{m-1} \in H$ such that:

- $v_{1}=v$;

- $v_{m}=w$;

- $\left\{v_{i}, v_{i+1}\right\} \subseteq h_{i}$ for each $i=1, \ldots, m-1$.

From here on, we fix a hypergraph with real coefficients $\Gamma=(V, H, \mathcal{C})$ on $N$ vertices $v_{1}, \ldots, v_{N}$ and $M$ hyperedges $h_{1}, \ldots, h_{M}$. For simplicity, assume that $\Gamma$ has no isolated vertices, i.e. we assume that each vertex is contained in at least one hyperedge, and we assume that $\Gamma$ is connected.

Changing the orientation of a hyperedge $h$ means substituting every $C_{v, h}$ by $-C_{v, h}$. We denote the two orientations by $(h,+)$ and $(h,-)$. Analogously to differential forms in Riemannian geometry [28] and to the case of chemical hypergraphs [4], we shall consider functions $\gamma: H \rightarrow \mathbb{R}$ that satisfy

$$
\gamma(h,-)=-\gamma(h,+),
$$


that is, changing the orientation of $h$ produces a minus sign.

Definition 2.5. Given $h \in H$, its cardinality, denoted $|h|$, is the number of vertices that are contained in $h$.

Definition 2.6. Given $v \in V$, its degree is

$$
\operatorname{deg} v:=\sum_{h \in H}\left(C_{v, h}\right)^{2} .
$$

The $N \times N$ diagonal degree matrix of $\Gamma$ is

$$
D:=\operatorname{diag}\left(\operatorname{deg} v_{i}\right)_{i=1, \ldots, N} .
$$

Remark 2.7. In literature, there are various ways of defining the degree of a vertex in a hypergraph. Our definition of vertex degree coincides with the one in [7] for chemical hypergraphs and it coincides, in particular, with the classical definition of degree in the case of graphs. Since it is natural to choose a vertex degree that is always non-negative, one could as well consider, for instance, $\sum_{h \in H}\left|C_{v, h}\right|$ as degree of a vertex $v$. However, here we choose to define the degree of $v$ as in (3) in order to be able, later on, to write the vertex normalized Laplacian in a matrix formulation that coincides with the one that is commonly used in literature for graphs (cf. Proposition 3.1 below).

Observe also that, since the coefficients $C_{v, h}$ are non-zero provided $v \in h$ and since we are assuming that there are no isolated vertices, there are no vertices of degree 0 . In particular, $D$ is an invertible matrix.

Definition 2.8. The $N \times N$ adjacency matrix of $\Gamma$ is $A:=\left(A_{i j}\right)_{i j}$, where $A_{i i}:=0$ for all $i=1, \ldots, N$ and

$$
A_{i j}:=-\sum_{h \in H} C_{v_{i}, h} \cdot C_{v_{j}, h} \quad \text { for all } i \neq j .
$$

Remark 2.9. In the case of simple graphs, $A$ coincides with the classical adjacency matrix that has $(0,1)$-entries and it's such that $A_{i, j}=1$ if and only if $v_{i}$ and $v_{j}$ are joined by an edge.

Definition 2.10. The $N \times M$ incidence matrix of $\Gamma$ is $\mathcal{I}:=\left(\mathcal{I}_{i j}\right)_{i j}$, where

$$
\mathcal{I}_{i j}:=C_{v_{i}, h_{j}} .
$$

Remark 2.11. Each row $\mathcal{I}_{i}$ of $\mathcal{I}$ represents a vertex $v_{i}$ and each column $\mathcal{I}^{j}$ of $\mathcal{I}$ represents a hyperedge $h_{j}$.

Definition 2.12. Given $J \subseteq\{1, \ldots, M\}$, we say that the hyperedges $\left\{h_{j}\right\}_{j \in J}$ are linearly independent if the corresponding columns in the incidence matrix are linearly independent, that is, if $\left\{\mathcal{I}^{j}\right\}_{j \in J}$ are linearly independent.

Remark 2.13. Linear dependence of hyperedges means the following: we see each hyperedge as the weighted sum of all its vertices, where the weights are the coefficients. If a hyperedge can be written as a linear combination of the other ones, we talk about linear dependence. 
Definition 2.14. Given $\omega, \gamma: H \rightarrow \mathbb{R}$, their scalar product is

$$
(\omega, \gamma)_{H}:=\sum_{h \in H} \omega(h) \cdot \gamma(h)
$$

Definition 2.15. Given $f, g: V \rightarrow \mathbb{R}$, their scalar product is

$$
(f, g)_{V}:=\sum_{v \in V} \operatorname{deg} v \cdot f(v) \cdot g(v)
$$

Remark 2.16. The scalar products defined above are both positive definite.

Definition 2.17. Given $f: V \rightarrow \mathbb{R}$ and $h \in H$, its boundary operator is

$$
\delta f(h):=\sum_{v \in V} C_{v, h} \cdot f(v)
$$

Remark 2.18. Observe that

$$
\delta:\{f: V \rightarrow \mathbb{R}\} \longrightarrow\{\gamma: H \rightarrow \mathbb{R}\}
$$

and, for each $f: V \rightarrow \mathbb{R}, \delta f$ satisfies (2).

Definition 2.19. Let

$$
\delta^{*}:\{\gamma: H \rightarrow \mathbb{R}\} \longrightarrow\{f: V \rightarrow \mathbb{R}\}
$$

be defined by

$$
\delta^{*}(\gamma)(v):=\frac{\sum_{h \in H} C_{v, h} \cdot \gamma(h)}{\operatorname{deg} v}
$$

Lemma 2.20. $\delta^{*}$ is such that $(\delta f, \gamma)_{H}=\left(f, \delta^{*} \gamma\right)_{V}$, therefore it is the (unique) adjoint operator of $\delta$.

Proof.

$$
\begin{aligned}
(\delta f, \gamma)_{H} & =\sum_{h \in H} \gamma(h) \cdot\left(\sum_{v \in V} C_{v, h} \cdot f(v)\right) \\
& =\sum_{v \in V} f(v) \cdot\left(\sum_{h \in H} C_{v, h} \cdot \gamma(h)\right) \\
& =\sum_{v \in V} \operatorname{deg} v \cdot f(v) \cdot \frac{\left(\sum_{h \in H} C_{v, h} \cdot \gamma(h)\right)}{\operatorname{deg} v} \\
& =\sum_{v \in V} \operatorname{deg} v \cdot f(v) \cdot \delta^{*}(\gamma)(v) \\
& =\left(f, \delta^{*} \gamma\right)_{V} .
\end{aligned}
$$


Definition 2.21. Given $f: V \rightarrow \mathbb{R}$ and given $v \in V$, let

$$
\begin{aligned}
L f(v): & =\delta^{*}(\delta f)(v) \\
& =\frac{\sum_{h \in H} C_{v, h} \cdot \delta f(h)}{\operatorname{deg} v} \\
& =\frac{\sum_{h \in H} C_{v, h} \cdot\left(\sum_{v^{\prime} \in V} C_{v^{\prime}, h} \cdot f\left(v^{\prime}\right)\right)}{\operatorname{deg} v} \\
& =\frac{1}{\operatorname{deg} v} \cdot\left(\sum_{h \in H} \sum_{v^{\prime} \in V} C_{v, h} \cdot C_{v^{\prime}, h} \cdot f\left(v^{\prime}\right)\right) \\
& =f(v)+\frac{1}{\operatorname{deg} v} \cdot\left(\sum_{h \in H} \sum_{v^{\prime} \in V \backslash\{v\}} C_{v, h} \cdot C_{v^{\prime}, h} \cdot f\left(v^{\prime}\right)\right) .
\end{aligned}
$$

Analogously, given $\gamma: H \rightarrow \mathbb{R}$ and $h \in H$, let

$$
\begin{aligned}
L^{H} \gamma(h): & =\delta\left(\delta^{*} \gamma\right)(h) \\
& =\sum_{v \in V} C_{v, h} \cdot \delta^{*} \gamma(v) \\
& =\sum_{v \in V} C_{v, h} \cdot\left(\frac{\sum_{h^{\prime} \in H} C_{v, h^{\prime}} \cdot \gamma\left(h^{\prime}\right)}{\operatorname{deg} v}\right) \\
& =\sum_{v \in V} \sum_{h^{\prime} \in H} \frac{1}{\operatorname{deg} v} \cdot C_{v, h} \cdot C_{v, h^{\prime}} \cdot \gamma\left(h^{\prime}\right) .
\end{aligned}
$$

We call $L$ and $L^{H}$ the vertex normalized Laplacian and the hyperedge normalized Laplacian, respectively. They coincide with the ones in [4] in the case when the coefficients are in $\{-1,0,+1\}$.

Remark 2.22. Note that the operators $L$ and $L^{H}$ do not change if we change the signs of all coefficients $C_{v, h}$ for any hyperedge $h$ and all vertices $v$ in that hyperedge. Moreover, the operator $L^{H}$ doesn't change if, for each $v \in V$ and each $h \in H$, we replace $C_{v, h}$ by $C_{v, h} \cdot a(v)$, where $a(v)$ is any real coefficient depending only on $v$.

\section{First properties}

We first observe that, as in the case of chemical hypergraphs [4],

- $L$ and $L^{H}$ are the two compositions of $\delta$ and $\delta^{*}$, which are adjoint to each other. Therefore they are both self-adjoint, which implies that their eigenvalues are real.

- $L$ and $L^{H}$ are non-negative operators. In fact, given $f: V \rightarrow \mathbb{R}$,

$$
\langle L f, f\rangle=\left\langle\delta^{*} \delta f, f\right\rangle=\langle\delta f, \delta f\rangle_{E} \geq 0 .
$$

Analogously, for $\gamma: E \rightarrow \mathbb{R}$,

$$
\left\langle L^{H} \gamma, \gamma\right\rangle_{E}=\left\langle\delta \delta^{*} \gamma, \gamma\right\rangle_{E}=\left\langle\delta^{*} \gamma, \delta^{*} \gamma\right\rangle \geq 0
$$

This implies that the eigenvalues of $L$ and $L^{H}$ are non-negative. 
- Since $L$ and $L^{H}$ are the two compositions of two linear operators, the non-zero eigenvalues of $L$ and $L^{H}$ are the same. In particular, if $f$ is an eigenfunction of $L$ with eigenvalue $\lambda \neq 0$, then $\delta f$ is an eigenfunction of $L^{H}$ with eigenvalue $\lambda$; if $\gamma$ is an eigenfunction of $L^{H}$ with eigenvalue $\lambda^{\prime} \neq 0$, then $\delta^{*} \gamma$ is an eigenfunction of $L$ with eigenvalue $\lambda^{\prime}$.

This also implies that the two operators only differ in the multiplicity of the eigenvalue 0 . Let $m_{V}$ and $m_{H}$ be the multiplicity of the eigenvalue 0 of $L$ and $L^{H}$, respectively. Then,

$$
m_{V}-m_{H}=N-M \text {. }
$$

Now, observe that we can see a function $f: V \rightarrow \mathbb{R}$ as a vector $\left(f_{1}, \ldots, f_{N}\right) \in \mathbb{R}^{N}$ such that $f_{i}=f\left(v_{i}\right)$ and, analogously, we can see a function $\gamma: H \rightarrow \mathbb{R}$ as a vector $\left(\gamma_{1}, \ldots, \gamma_{M}\right) \in \mathbb{R}^{M}$ such that $\gamma_{k}=\gamma\left(h_{k}\right)$. In view of this observation, we can write $L$ and $L^{H}$ in a matrix form.

In the following, we denote by Id the $N \times N$ identity matrix.

Proposition 3.1. The normalized Laplacians can be rewritten in matrix form as

$$
L=\mathrm{Id}-D^{-1} A \quad \text { and } \quad L^{H}=\mathcal{I}^{\top} D^{-1} \mathcal{I} .
$$

Proof. Observe that the $(i j)$-entry of the $N \times N$ matrix Id $-D^{-1} A$ is

$$
\left(\operatorname{Id}-D^{-1} A\right)_{i j}= \begin{cases}1 & \text { if } i=j \\ -\frac{A_{i j}}{\operatorname{deg} v_{i}} & \text { if } i \neq j .\end{cases}
$$

Therefore, given $f: V \rightarrow \mathbb{R}$ that can be seen as a vector $f=\left(f_{1}, \ldots, f_{N}\right) \in \mathbb{R}^{N}$,

$$
\begin{aligned}
\left(\left(\operatorname{Id}-D^{-1} A\right) \cdot f\right)_{i} & =f_{i}-\frac{1}{\operatorname{deg} v_{i}} \cdot \sum_{j \neq i, j=1}^{N} A_{i j} \cdot f_{j} \\
& =f\left(v_{i}\right)+\frac{1}{\operatorname{deg} v_{i}} \cdot\left(\sum_{h \in H} \sum_{v_{j} \in V \backslash\left\{v_{i}\right\}} C_{v_{i}, h} \cdot C_{v_{j}, h} \cdot f\left(v_{j}\right)\right) \\
& =L f\left(v_{i}\right) .
\end{aligned}
$$

Similarly, the $(k l)$-entry of the $M \times M$ matrix $\mathcal{I}^{\top} D^{-1} \mathcal{I}$ is

$$
\left(\mathcal{I}^{\top} D^{-1} \mathcal{I}\right)_{k l}=\sum_{i=1}^{N} \frac{1}{\operatorname{deg} v_{i}} \cdot \mathcal{I}_{i k} \cdot \mathcal{I}_{i l}
$$

Therefore, given $\gamma: H \rightarrow \mathbb{R}$ that can be seen as a vector $\gamma=\left(\gamma_{1}, \ldots, \gamma_{M}\right) \in \mathbb{R}^{M}$,

$$
\begin{aligned}
\left(\left(\mathcal{I}^{\top} D^{-1} \mathcal{I}\right) \cdot \gamma\right)_{k} & =\sum_{i=1}^{N} \sum_{l=1}^{M} \frac{1}{\operatorname{deg} v_{i}} \cdot \mathcal{I}_{i k} \mathcal{I}_{i l} \cdot \gamma_{l} \\
& =\sum_{v_{i} \in V} \sum_{h_{l} \in H} \frac{1}{\operatorname{deg} v_{i}} \cdot C_{v_{i}, h_{k}} \cdot C_{v_{i}, h_{l}} \cdot \gamma\left(h_{l}\right) \\
& =L^{H} \gamma\left(h_{k}\right) .
\end{aligned}
$$


Corollary 3.2. The sum of the eigenvalues of $L$ (and $L^{H}$ ) equals $N$.

Proof. It follows from Proposition 3.1, since the sum of a matrix's eigenvalues equals its trace, and the trace of $L$ is $N$.

From here on, we will arrange the $N$ eigenvalues of $L$ as

$$
0 \leq \lambda_{1} \leq \ldots \leq \lambda_{N} \leq N
$$

and we will arrange the $M$ eigenvalues of $L^{H}$ as

$$
0 \leq \lambda_{1}^{H} \leq \ldots \leq \lambda_{M}^{H} \leq N .
$$

We say that the eigenvalues of $L$ are the spectrum of $\Gamma$.

Remark 3.3. Assuming the connectivity of $\Gamma$ is not restrictive. In fact, it is clear by definition of $L$ that the spectrum of a disconnected hypergraph is equal to the union of the spectra of its connected components.

\section{The eigenvalue 0}

By (4), a function $f$ on the vertex set satisfies $L f=0$ if and only if, for every $h \in H$,

$$
\sum_{v \in V} C_{v, h} \cdot f(v)=0
$$

Thus, to create an eigenvalue 0 of $L$, we need a function $f: V \rightarrow \mathbb{R}$ such that is not identically 0 and satisfies (6).

Similarly, by (5), in order to get an eigenvalue 0 of $L^{H}$, we need $\gamma: H \rightarrow \mathbb{R}$ that is not identically 0 , satisfying (2) and

$$
\sum_{h \in H} C_{v, h} \cdot \gamma(h)=0
$$

for every vertex $v$.

The following results generalize the ones in [4, Section 5].

\section{Proposition 4.1.}

$$
\operatorname{dim}(\operatorname{ker} \mathcal{I})=m_{H} \quad \text { and } \quad \operatorname{dim}\left(\operatorname{ker} \mathcal{I}^{\top}\right)=m_{V} .
$$

Proof. Fix a function $\gamma: H \rightarrow \mathbb{R}$ that we can see as a vector $\left(\gamma_{1}, \ldots, \gamma_{M}\right) \in \mathbb{R}^{M}$. Then,

$$
\begin{aligned}
\mathcal{I} \cdot\left(\begin{array}{c}
\gamma_{1} \\
\vdots \\
\gamma_{M}
\end{array}\right)=\mathbf{0} & \Longleftrightarrow \sum_{j=1}^{M} \mathcal{I}_{i j} \cdot \gamma_{j}=0 \quad \forall i \in\{1, \ldots, N\} \\
& \Longleftrightarrow \sum_{j=1}^{M} C_{v_{i}, h_{j}} \cdot \gamma\left(h_{j}\right)=0 \quad \forall i \in\{1, \ldots, N\} \\
& \Longleftrightarrow \gamma \text { is an eigenfunction of } L^{H} \text { with eigenvalue } 0 .
\end{aligned}
$$

Therefore $\operatorname{dim}(\operatorname{ker} \mathcal{I})=m_{H}$ and the proof of the second equality is analogous. 


\section{Corollary 4.2.}

$$
m_{H}=M-\text { maximum number of linearly independent hyperedges }
$$

and

$$
m_{V}=N-\text { maximum number of linearly independent hyperedges. }
$$

Proof. It follows by Proposition 4.1 and by the Rank-Nullity Theorem.

Remark 4.3. In the case when the coefficients are integers, the equation

$$
\mathcal{I} \cdot\left(\begin{array}{c}
\gamma_{1} \\
\vdots \\
\gamma_{M}
\end{array}\right)=\mathbf{0}
$$

for the eigenfunctions $\gamma$ of $L^{H}$ that have eigenvalue 0 , coincides with the metabolite balancing equation [29]. In the setting of metabolic pathway analysis, the $v_{i}$ 's represent metabolites, the $h_{j}$ 's represent metabolic reactions, and a vector $\gamma=\left(\gamma_{1}, \ldots, \gamma_{M}\right)$ is a flux distribution. A solution of (8) represents a balance between the consumed metabolites and produced metabolites.

Furthermore, in the metabolic pathway analysis, an elementary flux mode (EFM) is defined by Equation (8) together with the following two conditions:

1. Feasibility: $\gamma(h) \geq 0$ if the reaction represented by $h \in H$ is irreversible. In our case, this condition is naturally satisfied because, by considering undirected hypergraphs, we are representing only reversible reactions (at least from the theoretical point of view) as we are considering both orientations for every hyperedge.

2. Non-decomposability: there is no non-zero vector $\left(\gamma_{1}^{\prime}, \ldots, \gamma_{M}^{\prime}\right) \in \mathbb{R}^{M}$ satisfying Equation (8) and the Feasibility condition such that

$$
P(\gamma):=\{h \in H: \gamma(h) \neq 0\} \supset P\left(\gamma^{\prime}\right) .
$$

This condition is also called genetic independence and, in our case, it corresponds to a specific choice of a basis for the kernel of $\mathcal{I}$.

\section{Min-max principle}

We can apply the Courant-Fischer-Weyl min-max principle in order to characterize all the eigenvalues of $L$ and $L^{H}$.

Theorem 5.1 (Courant-Fischer-Weyl min-max principle). Let $W$ be an n-dimensional vector space with a positive definite scalar product $(.,$.$) . Let \mathcal{W}_{k}$ be the family of all $k$-dimensional subspaces of $W$. Let $B: W \rightarrow W$ be a self-adjoint linear operator. Then the eigenvalues $\mu_{1} \leq \ldots \leq \mu_{n}$ of $B$ can be obtained by

$$
\mu_{k}=\min _{W_{k} \in \mathcal{W}_{k}} \max _{g(\neq 0) \in W_{k}} \frac{(B g, g)}{(g, g)}=\max _{W_{n-k+1} \in \mathcal{W}_{n-k+1}} \min _{g(\neq 0) \in W_{n-k+1}} \frac{(B g, g)}{(g, g)} .
$$


The vectors $g_{k}$ realizing such a min-max or max-min then are corresponding eigenvectors, and the min-max spaces $\mathcal{W}_{k}$ are spanned by the eigenvectors for the eigenvalues $\mu_{1}, \ldots, \mu_{k}$, and analogously, the max-min spaces $\mathcal{W}_{n-k+1}$ are spanned by the eigenvectors for the eigenvalues $\mu_{k}, \ldots, \mu_{n}$. Thus, we also have

$$
\mu_{k}=\min _{g \in W,\left(g, g_{j}\right)=0 \text { for } j=1, \ldots, k-1} \frac{(B g, g)}{(g, g)}=\max _{g \in V,\left(g, g_{l}\right)=0 \text { for } l=k+1, \ldots, n} \frac{(B g, g)}{(g, g)} .
$$

In particular,

$$
\mu_{1}=\min _{g \in W} \frac{(B g, g)}{(g, g)}, \quad \mu_{n}=\max _{g \in W} \frac{(B g, g)}{(g, g)} .
$$

Definition 5.2. $\frac{(B g, g)}{(g, g)}$ is the Rayleigh quotient of $g$.

Remark 5.3. Without loss of generality, we may assume $(g, g)=1$ in (9).

As a consequence of the min-max principle, we can write the eigenvalues of $L$ and $L^{H}$ as the min-max or max-min of the Rayleigh quotients

$$
\begin{aligned}
\operatorname{RQ}(f):= & \frac{(\delta f, \delta f)_{H}}{(f, f)_{V}} \\
& =\frac{\sum_{h \in H}\left(\sum_{v \in V} C_{v, h} \cdot f(v)\right)^{2}}{\sum_{v \in V} \operatorname{deg} v \cdot f(v)^{2}}, \quad \text { for } f: V \rightarrow \mathbb{R}
\end{aligned}
$$

and

$$
\begin{aligned}
\operatorname{RQ}(\gamma): & =\frac{\left(\delta^{*} \gamma, \delta^{*} \gamma\right)_{V}}{(\gamma, \gamma)_{H}} \\
& =\frac{\sum_{v \in V} \frac{1}{\operatorname{deg} v} \cdot\left(\sum_{h \in H} C_{v, h} \cdot \gamma(h)\right)^{2}}{\sum_{h \in H} \gamma(h)^{2}}, \text { for } \gamma: H \rightarrow \mathbb{R} .
\end{aligned}
$$

In particular,

$$
\lambda_{1}=\lambda_{1}^{H}=\min _{f: V \rightarrow \mathbb{R}} \operatorname{RQ}(f)=\min _{\gamma: H \rightarrow \mathbb{R}} \operatorname{RQ}(\gamma)
$$

and

$$
\lambda_{N}=\lambda_{M}^{H}=\max _{f: V \rightarrow \mathbb{R}} \operatorname{RQ}(f)=\max _{\gamma: H \rightarrow \mathbb{R}} \operatorname{RQ}(\gamma) .
$$

\section{Bipartiteness and largest eigenvalue}

A simple graph is bipartite if there exists a bipartition of the vertex set into two disjoint sets $V=V_{2} \sqcup V_{2}$ such that each edge in is between a vertex in $V_{1}$ and a vertex in $V_{2}$. Bipartiteness is an important geometrical property and it is known that the largest eigenvalue of a graph measures how far the graph is from being bipartite. In particular, as shown by Chung in [5], $\lambda_{N} \leq 2$ for any simple connected graph $\Gamma$, with equality if and only if $\Gamma$ is bipartite. 


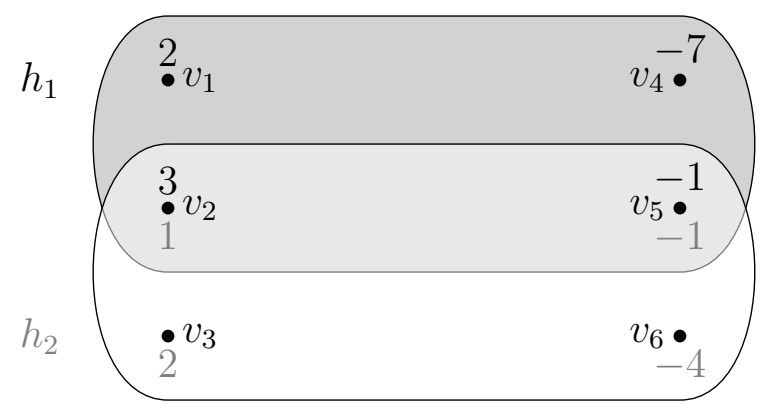

Figure 1: A bipartite hypergraph with $V_{1}=\left\{v_{1}, v_{2}, v_{3}\right\}$ and $V_{2}=\left\{v_{4}, v_{5}, v_{6}\right\}$. The numbers near the vertices represent the corresponding coefficients.

For chemical hypergraphs the notion of bipartiteness has been generalized in [4], and in [7] it has been proved that

$$
\lambda_{N} \leq \max _{h \in H}|h|,
$$

with equality if and only if $\Gamma$ is bipartite and $|h|$ is constant for all $h$. Hence, for a chemical hypergraph with constant $|h|$, we can say that $|h|-\lambda_{N}$ estimates how different the hypergraph is from being bipartite.

Here we prove a further generalization of the above inequality to the case of hypergraphs with real coefficients. Before, we generalize the definition of bipartite hypergraph.

Definition 6.1. Given $h \in H$ and $v \in h, v$ is an input (resp. output) for $h$ if $C_{v, h}>0$ (resp. $C_{v, h}<0$ ).

Definition 6.2. $\Gamma$ is bipartite if one can decompose the vertex set as a disjoint union $V=V_{1} \sqcup V_{2}$ such that, for every $h \in H$, either $h$ has all its inputs in $V_{1}$ and all its outputs in $V_{2}$, or vice versa (Figure 1 ).

Theorem 6.3. For every hypergraph with real coefficients $\Gamma$,

$$
\lambda_{N} \leq \max _{h \in H}|h|,
$$

with equality if and only if $\Gamma$ is bipartite, $|h|$ is constant for all $h \in H$ and it is possible to define a non-zero function $f$ on the vertex set such that

$$
g(h):=\left|C_{v, h} \cdot f(v)\right|
$$

does not depend on $v$, for all $h \in H$ and $v \in h$. In this case, $f$ satisfying (11) is an eigenfunction for $\lambda_{N}$.

Proof. Let $f: V \rightarrow \mathbb{R}$ be an eigenfunction for $\lambda_{N}$. By the min-max principle,

$$
\lambda_{N}=\frac{\sum_{h \in H}\left(\sum_{v \in V} C_{v, h} \cdot f(v)\right)^{2}}{\sum_{v \in V} \operatorname{deg} v f(v)^{2}}
$$




$$
\leq \frac{\sum_{h \in H}\left(\sum_{v \in V}\left|C_{v, h} \cdot f(v)\right|\right)^{2}}{\sum_{v \in V} \operatorname{deg} v f(v)^{2}},
$$

with equality if and only if $f$ has its non-zero values on a bipartite sub-hypergraph. Now, for each $h \in H$,

$$
\begin{aligned}
\left(\sum_{v \in V}\left|C_{v, h} \cdot f(v)\right|\right)^{2} & =\sum_{v \in h} C_{v, h}^{2} \cdot f(v)^{2}+\sum_{\{v, w\}: v \neq w \in h} 2 \cdot\left|C_{v, h} \cdot f(v)\right| \cdot\left|C_{w, h} \cdot f(w)\right| \\
& \leq \sum_{v \in h} C_{v, h}^{2} \cdot f(v)^{2}+\sum_{\{v, w\}: v \neq w \in h}\left(C_{v, h}^{2} \cdot f(v)^{2}+C_{w, h}^{2} \cdot f(w)^{2}\right) \\
& =\sum_{v \in h} C_{v, h}^{2} \cdot f(v)^{2}+\sum_{v \in h}(|h|-1) \cdot C_{v, h}^{2} \cdot f(v)^{2} \\
& =|h| \cdot \sum_{v \in h} C_{v, h}^{2} \cdot f(v)^{2},
\end{aligned}
$$

with equality if and only if $\left|C_{v, h} \cdot f(v)\right|=: g(h)$ is constant for all $v \in h$. Therefore,

$$
\begin{aligned}
\frac{\sum_{h \in H}\left(\sum_{v \in V}\left|C_{v, h} \cdot f(v)\right|\right)^{2}}{\sum_{v \in V} \operatorname{deg} v f(v)^{2}} & \leq \frac{\sum_{h \in H}|h| \cdot \sum_{v \in h} C_{v, h}^{2} \cdot f(v)^{2}}{\sum_{v \in V} \operatorname{deg} v f(v)^{2}} \\
& =\frac{\sum_{v \in V} \sum_{h \ni v}|h| \cdot C_{v, h}^{2} \cdot f(v)^{2}}{\sum_{v \in V} \operatorname{deg} v f(v)^{2}} \\
& \leq\left(\max _{h \in H}|h|\right) \cdot \frac{\sum_{v \in V} f(v)^{2}\left(\sum_{h \ni v} C_{v, h}^{2}\right)}{\sum_{v \in V} \operatorname{deg} v f(v)^{2}} \\
& =\left(\max _{h \in H}|h|\right) \cdot \frac{\sum_{v \in V} \operatorname{deg} v f(v)^{2}}{\sum_{v \in V} \operatorname{deg} v f(v)^{2}} \\
& =\max _{h \in H}|h|,
\end{aligned}
$$

where the first inequality is an equality if and only if $g(h)=\left|C_{v, h} \cdot f(v)\right|$ does not depend on $v$, for all $h \in H$ and $v \in h$, and the last inequality is an equality if and only if $|h|$ is constant for all $h$. Putting everything together, we have that

$$
\lambda_{N} \leq \max _{h \in H}|h|,
$$

with equality if and only if $|h|$ is constant for all $|h|, f$ is defined on a bipartite sub-hypergraph and

$$
f(v)=\frac{g(h)}{\left|C_{v, h}\right|}, \quad \forall v \in V \text { and } h \ni v .
$$

Now, in the case when equality is achieved, assume by contradiction that there exists $v^{\prime} \in V$ with $f\left(v^{\prime}\right)=0$. Then, by (12) and by the connectivity of $\Gamma, f(v)=0$ for all $v \in V$. This is a contradiction. Therefore, if $f$ satisfies (12) and it's defined on a bipartite sub-hypergraph, then $\Gamma$ itself is bipartite. This proves the claim. 


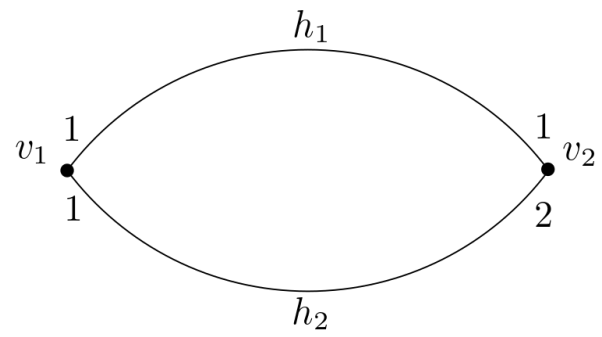

Figure 2: The hypergraph in Example 6.5.

Remark 6.4. If $\Gamma$ is bipartite, $h$ is constant for all $h \in H$ and $\left|C_{v, h}\right|$ is constant for all $v \in V$ and $h \in H$, then clearly it is always possible to find a function $f$ that satisfies (11). Therefore, in this case, by Theorem 6.3, $\lambda_{N}=\max _{h \in H}|h|$ and $f$ is a corresponding eigenfunction. However, it is not always possible to find a function $f$ that satisfies (11), as shown by the next example.

Example 6.5. Let $\Gamma=(V, H, \mathcal{C})$ be such that (Figure 2):

- $V=\left\{v_{1}, v_{2}\right\}$

- $H=\left\{h_{1}, h_{2}\right\}$,

- $C_{v_{1}, h_{1}}=C_{v_{2}, h_{1}}=C_{v_{1}, h_{2}}=1$, and

- $C_{v_{2}, h_{2}}=2$.

Then, $\Gamma$ is a connected bipartite hypergraph such that $|h|=2$ is constant for all $h \in H$. However, it is not possible to find a function $f$ that satisfies (11) and therefore, by Theorem 6.3, $\lambda_{N}=\lambda_{2}<2$. In fact, a function $f$ satisfying (11) in this case should be such that

- $\left|f\left(v_{1}\right)\right|=\left|f\left(v_{2}\right)\right|=g\left(h_{1}\right)$ and

- $\left|f\left(v_{1}\right)\right|=2 \cdot\left|f\left(v_{2}\right)\right|=g\left(h_{2}\right)$,

which implies $f\left(v_{1}\right)=f\left(v_{2}\right)=0$, but this is a contraction. In this case, in particular, the vertex normalized Laplacian is

$$
\begin{aligned}
L=\mathrm{Id} & -D^{-1} A \\
& =\left(\begin{array}{ll}
1 & 0 \\
0 & 1
\end{array}\right)-\left(\begin{array}{cc}
1 / 2 & 0 \\
0 & 1 / 5
\end{array}\right)\left(\begin{array}{cc}
0 & -3 \\
-3 & 0
\end{array}\right) \\
& =\left(\begin{array}{cc}
1 & 3 / 2 \\
3 / 5 & 1
\end{array}\right) .
\end{aligned}
$$

Its eigenvalues are

$$
\lambda_{1}=1-\frac{3}{\sqrt{10}} \cong 0.05 \text { and } \quad \lambda_{2}=1+\frac{3}{\sqrt{10}} \cong 1.95,
$$

with eigenvectors

$$
f_{1}=(\sqrt{5 / 2}, 1) \text { and } f_{2}=(-\sqrt{5 / 2}, 1)
$$

respectively. 


\section{Bounds on general eigenvalues}

In this section we give some general bounds on all eigenvalues of $\Gamma$.

Definition 7.1. Given $\hat{v} \in V$, we let $\Gamma \backslash \hat{v}:=(\hat{V}, \hat{H}, \hat{\mathcal{C}})$, where:

- $\hat{V}=V \backslash \hat{v}$,

- $\hat{H}=\{h \backslash \hat{v}: h \in H\}$, and

- $\hat{\mathcal{C}}=\left\{C_{v, h} \in \mathcal{C}: v \in \hat{V}\right.$ and $\left.h \in \hat{H}\right\}$.

We say that $\Gamma \backslash \hat{v}$ is obtained from $\Gamma$ by a weak vertex deletion of $\hat{v}$. We say that $\Gamma$ is obtained from $\Gamma \backslash \hat{v}$ by a weak vertex addition of $\hat{v}$.

Lemma 7.2. If $\hat{\Gamma}$ is obtained from $\Gamma$ by weak-deleting $r$ vertices,

$$
\lambda_{k}(\Gamma) \leq \lambda_{k}(\hat{\Gamma}) \leq \lambda_{k+r}(\Gamma) \quad \text { for all } k \in\{1, \ldots, n-r\} .
$$

Proof. As in the proof of Lemma 2.20 in [8], the claim follows directly by inductively applying the Cauchy Interlacing Theorem [30, Theorem 4.3.17].

Theorem 7.3. Up to a relabelling of the hyperedges $h_{1}, \ldots, h_{M}$, assume that

$$
\left|h_{1}\right| \leq \ldots \leq\left|h_{M}\right|
$$

Then,

$$
\lambda_{N-1} \leq \max \left\{\left|h_{M}\right|-1,\left|h_{M-1}\right|\right\}
$$

and, more generally,

$$
\lambda_{N-k} \leq \max \left\{\left|h_{M}-k\right|,\left|h_{M-1}\right|-k+1, \ldots,\left|h_{M-k}\right|\right\}=\max \left\{\left|h_{M-i}\right|-k+i\right\}_{i=0, \ldots, k}
$$

for all $k \in\{1, \ldots, N-1\}$.

Proof. Fix a vertex $v \in h_{M}$. Then, the largest hyperedge cardinality of $\Gamma \backslash v$ is either $\left|h_{M}\right|-1$ or $\left|h_{M-1}\right|$. Therefore, by Theorem 6.3 applied to $\Gamma \backslash v$,

$$
\lambda_{N-1}(\Gamma \backslash v) \leq \max \left\{\left|h_{M}\right|-1,\left|h_{M-1}\right|\right\} .
$$

Together with Lemma 7.2, this proves (13). (14) follows by repeatedly applying (13).

Theorem 7.4. Let $\lambda_{\min }$ be the smallest non-zero eigenvalue of $\Gamma$. Then,

$$
\lambda_{\min } \leq \frac{N}{N-m_{V}}=\frac{N}{M-m_{H}} \leq \lambda_{N}
$$

and one of the above inequalities is an equality if and only if $\lambda_{\min }=\lambda_{N}$.

Proof. Same as the proof of Theorem 6.5 in 8 . 
Remark 7.5. By Theorem 6.3 and Theorem 7.4 we have that

$$
\frac{N}{N-m_{V}}=\frac{N}{M-m_{H}} \leq \lambda_{N} \leq \max _{h \in H}|h| .
$$

Hence

$$
m_{V} \leq N\left(1-\frac{1}{\max _{h \in H}|h|}\right)
$$

and

$$
m_{H} \leq M-\frac{N}{\max _{h \in H}|h|}
$$

We can therefore estimate $\max _{h \in H}|h|$ with $m_{V}$ or $m_{H}$, and vice versa.

\section{Symmetries}

It is known that symmetries can leave signatures in the spectrum of a graph or a hypergraph [13, 31 33]. For instance,

- Two vertices in a simple graph are called duplicate if they have the same neighbors and it is well known that $\hat{n}$ duplicate vertices produce the eigenvalue 1 with multiplicity at least $\hat{n}-1$ [34];

- More generally, two vertices in a chemical hypergraph are called duplicate if the corresponding rows/columns of the adjacency matrix are the same and it is still true that $\hat{n}$ duplicate vertices produce the eigenvalue 1 with multiplicity at least $\hat{n}-1$ [8];

- Two vertices in a chemical hypergraph are called twin if they belong exactly to the same hyperedges with the same signs, and $\hat{n}$ twin vertices produce the eigenvalue 0 with multiplicity at least $\hat{n}-1[9]$.

Here we generalize the definitions of twin and anti-twin vertices to the case of hypergraphs with real coefficients, and we show the effect of various hypergraph symmetries in the spectrum of the normalized Laplacian. The propositions in this sections contain results that are new also for the case of chemical hypergraphs.

Definition 8.1. Two distinct vertices $v_{i}$ and $v_{j}$ are:

- Twin, if $C_{v_{i}, h}=C_{v_{j}, h}$ for all $h \in H$;

- Anti-twin, if $C_{v_{i}, h}=-C_{v_{j}, h}$ for all $h \in H$;

- Duplicate, if if the corresponding rows/columns of the adjacency matrix are the same;

- Anti-duplicate, if the corresponding rows (equivalently, columns) of the adjacency matrix have opposite sign. 
Remark 8.2. If $v_{i}$ and $v_{j}$ are twin vertices, then

$$
\operatorname{deg} v_{i}=\operatorname{deg} v_{j}=-A_{i j}
$$

while, if they are anti-twin,

$$
\operatorname{deg} v_{i}=\operatorname{deg} v_{j}=A_{i j}
$$

If $v_{i}$ and $v_{j}$ are duplicate or anti-duplicate vertices, their degrees are not necessarily the same and $A_{i j}=A_{i i}=A_{j j}=0$.

We need a preliminary remark and a lemma in order to prove the propositions in this section.

Remark 8.3. $\lambda$ is an eigenvalue for $L$ and $f: V \rightarrow \mathbb{R}$ is a corresponding eigenfunction if and only if, for each $v_{i} \in V$, we have that

$$
\lambda \cdot f\left(v_{i}\right)=L f\left(v_{i}\right)=f\left(v_{i}\right)-\frac{1}{\operatorname{deg} v_{i}} \sum_{j \neq i} A_{i j} f\left(v_{j}\right),
$$

which can be rewritten as

$$
(1-\lambda) \cdot f\left(v_{i}\right)=\frac{1}{\operatorname{deg} v_{i}} \sum_{j \neq i} A_{i j} f\left(v_{j}\right) .
$$

Lemma 8.4. For all $v_{i}, v_{j} \in V$,

$$
\operatorname{deg} v_{i}+\operatorname{deg} v_{j} \geq \pm 2 A_{i j}
$$

with equality if and only if $C_{v_{i}, h}=\mp C_{v_{j}, h}$ for all $h \in H$.

Proof. We use the fact that, given $a, b \in \mathbb{R}, a^{2}+b^{2} \geq \mp 2 a b$, with equality if and only if $a=\mp b$. This implies that

$$
\sum_{h \in H}\left(C_{v_{i}, h}^{2}+C_{v_{j}, h}^{2}\right) \geq \mp 2 \cdot \sum_{h \in H} C_{v_{i}, h} \cdot C_{v_{j}, h},
$$

that is,

$$
\operatorname{deg} v_{i}+\operatorname{deg} v_{j} \geq \pm 2 \cdot A_{i j},
$$

with equality if and only if $C_{v_{i}, h}=\mp C_{v_{j}, h}$ for all $h \in H$.

Proposition 8.5. The constant functions are eigenfunctions corresponding to some eigenvalue $\lambda$ if and only if $\frac{1}{\operatorname{deg} v_{i}} \sum_{j \neq i} A_{i j}$ is constant for all $v_{i} \in V$. In this case,

$$
\lambda=1-\frac{1}{\operatorname{deg} v_{i}} \sum_{j \neq i} A_{i j} .
$$

Remark 8.6. In the case of simple graphs, $\sum_{j \neq i} A_{i j}=\operatorname{deg} v_{i}$ for each $v_{i} \in V$. Therefore, Proposition 8.5 applied to simple graphs states that $\lambda=0$ is always an eigenvalue, and that the constants are corresponding eigenfunctions. This is a well known result. 
Remark 8.7. In the case when $|h|$ is constant for all $h \in H$ and $C_{v, h}=1$ for all $h \in H$ and $v \in H$, the quantity in Proposition 8.5 is

$$
1-\frac{1}{\operatorname{deg} v_{i}} \sum_{j \neq i} A_{i j}=1-\frac{1}{\operatorname{deg} v_{i}} \sum_{h \ni v_{i}}(|h|-1)=|h|,
$$

for all $v_{i} \in V$. Proposition 8.5 tells us that, in this case, $|h|$ is an eigenvalue with constant eigenfunctions. From [7, Theorem 3.1], that we generalized in Theorem 6.3, we know that $\lambda=|h|$ is the largest eigenvalue in this case. Therefore, while for simple graphs the constants are eigenfunctions for $\lambda_{1}=0$, in the general case the constants can be eigenfunctions for larger eigenvalues.

Proof of Proposition 8.5. By (15), if $f$ is constant then

$$
1-\lambda=\frac{1}{\operatorname{deg} v_{i}} \sum_{j \neq i} A_{i j}
$$

for all $v_{i} \in V$. Vice versa, if $\frac{1}{\operatorname{deg} v_{i}} \sum_{j \neq i} A_{i j}$ is constant for all $v_{i} \in V$, then clearly the constant functions satisfy 15ith $\lambda=1-\frac{1}{\operatorname{deg} v_{i}} \sum_{j \neq i} A_{i j}$.

Proposition 8.8. A function $g: V \rightarrow \mathbb{R}$ such that $g\left(v_{i}\right)=g\left(v_{j}\right)=1$ for some $v_{i}, v_{j} \in V$ and $g\left(v_{k}\right)=0$ for all $k \neq i, j$ is an eigenfunction for some eigenvalue $\lambda$ if and only if:

- Either $\operatorname{deg} v_{i}=\operatorname{deg} v_{j}$ or $A_{i j}=0$;

- $A_{i k}=-A_{j k}$ for all $k \neq i, j$.

In this case,

$$
\lambda=1-\frac{A_{i j}}{\operatorname{deg} v_{i}} \leq 1
$$

and $\lambda=0$ if and only if $v_{i}$ and $v_{j}$ are anti-twin, while $\lambda=1$ if and only if $v_{i}$ and $v_{j}$ are anti-duplicate vertices.

Proof. By (15), if $g$ is an eigenfunction for $\lambda$, then

- $(1-\lambda) g\left(v_{i}\right)=(1-\lambda)=\frac{A_{i j}}{\operatorname{deg} v_{i}}$,

- $(1-\lambda) g\left(v_{j}\right)=(1-\lambda)=\frac{A_{i j}}{\operatorname{deg} v_{j}}$, and

- $(1-\lambda) g\left(v_{k}\right)=0=\frac{A_{i k}+A_{j k}}{\operatorname{deg} v_{k}}$ for all $k \neq i, j$.

Therefore, either $\operatorname{deg} v_{i}=\operatorname{deg} v_{j}$ or $A_{i j}=0 ; A_{i k}=-A_{j k}$ for all $k \neq i, j$, and $\lambda=1-\frac{A_{i j}}{\operatorname{deg} v_{i}}$.

Vice versa, if either $\operatorname{deg} v_{i}=\operatorname{deg} v_{j}$ or $A_{i j}=0$, and additionally $A_{i k}=-A_{j k}$ for all $k \neq i, j$, it is clear that $g$ satisfies 15 with $\lambda=1-\frac{A_{i j}}{\operatorname{deg} v_{i}}$.

In this case, Lemma 8.4 implies that $A_{i j} \leq \operatorname{deg} v_{i}$ which, on its turn, implies that $\lambda=1-\frac{A_{i j}}{\operatorname{deg} v_{i}} \leq 1$. Also, $\lambda=0$ if and only if $A_{i j}=\operatorname{deg} v_{i}$, therefore (again by Lemma 8.4 if and only if $C_{v_{i}, h}=-C_{v_{j}, h}$ for all $h \in H . \lambda=1$ if and only if $A_{i j}=0$. 


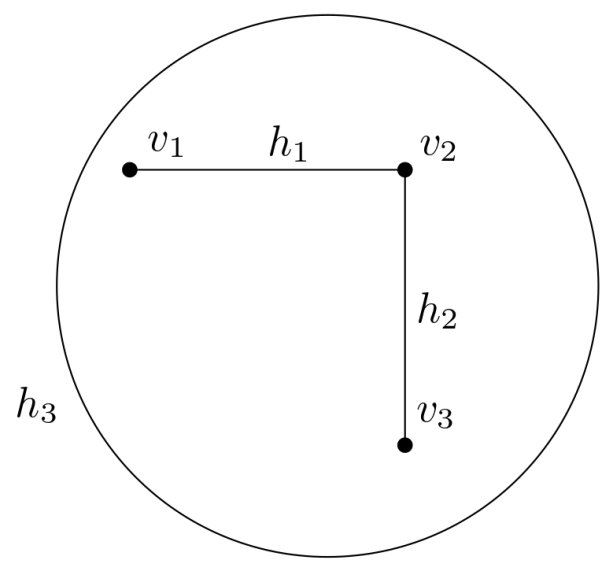

Figure 3: The hypergraph in Example 3.

Proposition 8.9. A function $f: V \rightarrow \mathbb{R}$ such that $f\left(v_{i}\right)=-f\left(v_{j}\right)=1$ for some $v_{i}, v_{j} \in V$ and $f\left(v_{k}\right)=0$ for all $k \neq i, j$ is an eigenfunction for some eigenvalue $\lambda$ if and only if:

- Either $\operatorname{deg} v_{i}=\operatorname{deg} v_{j}$ or $A_{i j}=0$;

- $A_{i k}=A_{j k}$ for all $k \neq i, j$.

In this case,

$$
\lambda=1+\frac{A_{i j}}{\operatorname{deg} v_{i}} \leq 1
$$

and $\lambda=0$ if and only if $v_{i}$ and $v_{j}$ are twin, while $\lambda=1$ if and only if $v_{i}$ and $v_{j}$ are duplicate vertices.

Proof. Analogous to the proof of Proposition 8.8 .

A direct consequence of Proposition 8.8 and Proposition 8.9 is

Corollary 8.10. If $\Gamma$ has $\tilde{n}$ duplicate (or anti-duplicate) vertices, then 1 is an eigenvalue with multiplicity at least $\tilde{n}-1$.

If $\Gamma$ has $\hat{n}$ twin (or anti-twin) vertices, then 0 is an eigenvalue with multiplicity at least $\hat{n}-1$.

We conclude with the example of a hypergraph that satisfies Proposition 8.9 for two vertices that are neither twin nor duplicate, but nevertheless are strongly symmetric.

Example 8.11. Let $\Gamma=(V, H, \mathcal{C})$ be such that (Figure 3):

- $V=\left\{v_{1}, v_{2}, v_{3}\right\}$ and $H=\left\{h_{1}, h_{2}\right\}$;

- $h_{1}=\left\{v_{1}, v_{2}\right\}, h_{2}=\left\{v_{2}, v_{3}\right\}$ and $h_{3}=\left\{v_{1}, v_{2}, v_{3}\right\}$

- All non-zero coefficients are equal 1. 
Then, $v_{1}$ and $v_{3}$ are neither twin nor duplicate vertices, but they satisfy the conditions of Proposition 8.9 since $\operatorname{deg} v_{1}=\operatorname{deg} v_{3}=2$ and $A_{12}=A_{32}=-2, A_{13}=-1$. By Proposition 8.9, this symmetry is reflected by the eigenvalue

$$
\lambda=1+\frac{A_{13}}{\operatorname{deg} v_{1}}=\frac{1}{2} .
$$

In particular, in this case

$$
\begin{aligned}
L=\mathrm{Id} & -D^{-1} A \\
& =\left(\begin{array}{lll}
1 & 0 & 0 \\
0 & 1 & 0 \\
0 & 0 & 1
\end{array}\right)-\left(\begin{array}{ccc}
1 / 2 & 0 & 0 \\
0 & 1 / 3 & 0 \\
0 & 0 & 1 / 2
\end{array}\right)\left(\begin{array}{ccc}
0 & -2 & -1 \\
-2 & 0 & -2 \\
-1 & -2 & 0
\end{array}\right) \\
& =\left(\begin{array}{ccc}
1 & 1 & 1 / 2 \\
2 / 3 & 1 & 2 / 3 \\
1 / 2 & 1 & 1
\end{array}\right) .
\end{aligned}
$$

Its eigenvalues are

$$
\lambda_{1}=\frac{15-\sqrt{201}}{12} \cong 0.07, \quad \lambda_{2}=\frac{1}{2} \quad \text { and } \quad \lambda_{3}=\frac{15+\sqrt{201}}{12} \cong 2.43 .
$$

\section{General symmetries}

Definition 9.1. An automorphism of the hypergraph $\Gamma=(V, H, \mathcal{C})$ consists of bijections $\sigma: V \rightarrow V$ and $\sigma: H \rightarrow H$ such that $\sigma(v) \in \sigma(h)$ iff $v \in h$ and for every $v \in V$ either $C_{\sigma(v), \sigma(h)}=C_{v, h}$ for all $h \in H$ or $=-C_{v, h}$ for all $h$. We may then put $s(\sigma)(v)=1$ in the first and $=-1$ in the second case. When $\sigma$ is clear, we simply write $s(v)$ in place of $s(\sigma)(v)$.

For a such an automorphism $\sigma$ and $f: V \rightarrow \mathbb{R}$, we put $\sigma_{*} f(v)=s(v) f(\sigma(v))$.

As we shall now verify, the Laplace operator commutes with automorphisms. This is trivial if $s(\sigma)(v)=1$ for all $v$. In the general case, we need to be careful with the signs.

Lemma 9.2. If $\sigma$ is an automorphism of the hypergraph $\Gamma=(V, H, \mathcal{C})$, then

$$
L\left(\sigma_{*} f\right)(v)=\sigma_{*}(L f)(v)
$$

for all $v \in V$ and $f: V \rightarrow \mathbb{R}$.

Proof.

$$
\begin{aligned}
L\left(\sigma_{*} f\right)(v) & =s(v) f(\sigma(v))+\frac{1}{\operatorname{deg} v} \cdot\left(\sum_{h \in H} \sum_{v^{\prime} \in V \backslash\{v\}} C_{v, h} \cdot C_{v^{\prime}, h} \cdot s\left(v^{\prime}\right) f\left(\sigma\left(v^{\prime}\right)\right)\right) \\
& =s(v) f(\sigma(v))+\frac{1}{\operatorname{deg} \sigma(v)} \cdot\left(\sum_{h \in H} \sum_{v^{\prime} \in V \backslash\{v\}} C_{\sigma(v), \sigma(h)} \cdot C_{\sigma\left(v^{\prime}\right), \sigma(h)} \cdot f\left(\sigma\left(v^{\prime}\right)\right)\right)
\end{aligned}
$$




$$
\begin{aligned}
& =s(v) f(\sigma(v))+\frac{1}{\operatorname{deg} \sigma(v)} \cdot\left(\sum_{h \in H} \sum_{v^{\prime} \in V \backslash\{v\}} \sigma(v) C_{v, h} \cdot C_{\sigma\left(v^{\prime}\right), \sigma(h)} \cdot f\left(\sigma\left(v^{\prime}\right)\right)\right) \\
& =\sigma_{*}(L f)(v) .
\end{aligned}
$$

Thus, the Laplacian $L$ commutes with hypergraph automorphisms $\sigma$. We can use Lemma 9.2 to decompose the spectrum of $L$. Let $\tau$ be an automorphism of the hypergraph $\Gamma=(V, H, \mathcal{C})$ with

$$
\tau^{2}=\mathrm{id}
$$

Then $\tau$ has two possible eigenvalues, \pm 1 , on the space of functions $f: V \rightarrow \mathbb{R}$, and $L$ leaves those two eigenspaces $L_{ \pm}$invariant. Also, $V=V_{0}+V_{1}$ where $\tau\left(v_{0}\right)=v_{0}$ precisely if $v_{0} \in V_{0}$. That is, $V_{0}$ is the set of those vertices that are fixed by $\tau$. Moreover, we can write $V_{1}=V^{\prime} \cup V^{\prime \prime}$ where $V^{\prime}, V^{\prime \prime}$ are disjoint and $\tau\left(V^{\prime}\right)=V^{\prime \prime}$. Since also $\tau\left(V^{\prime \prime}\right)=V^{\prime}$ because of (18), $V^{\prime}$ and $V^{\prime \prime}$ play symmetric roles.

W.l.o.g., we assume that $V^{\prime}$ (and hence also $V^{\prime \prime}$ ) is connected, as otherwise we can rearrange the decomposition of $V_{1}$ and/or write $\tau$ as the composition of several such automorphisms.

Lemma 9.3. In this situation, there is a $\left|V^{\prime}\right|$-dimensional space of functions $f$ : $V \rightarrow \mathbb{R}$ consisting of eigenfunctions of $L$ that vanish on $V_{0}$ and that are antisymmetric on $V^{\prime}$ and $V^{\prime \prime}$, that is, $f\left(v^{\prime \prime}\right)=-s\left(v^{\prime}\right) f\left(v^{\prime}\right)$ if $v^{\prime \prime}=\tau\left(v^{\prime}\right) \in V^{\prime \prime}$ for $v^{\prime} \in V^{\prime}$, and a remaining $\left(\left|V^{\prime}\right|+\left|V_{0}\right|\right)$-dimensional space of eigenfunctions that are symmetric on $V^{\prime}$ and $V^{\prime \prime}$, that is, $f\left(v^{\prime \prime}\right)=s\left(v^{\prime}\right) f\left(v^{\prime}\right)$.

Proof. The first class of functions are those that are eigenfunctions of $\tau$ for the eigenvalue -1 , where the second class has eigenvalue 1. By Lemma 9.2, these are unions of eigenspaces of $L$. Since $\left|V^{\prime \prime}\right|=\left|V^{\prime}\right|$ and $V=V_{0} \cup V^{\prime} \cup V^{\prime \prime}$, this generates the space of all functions on $V$.

Definition 9.4. Let $\Gamma=(V, H, \mathcal{C})$ be a hypergraph. An induced subhypergraph $\hat{\Gamma}$ has some nonempty vertex set $\hat{V} \subset V$ and a hyperedge set $\hat{H} \subset H$ such that any two $v_{1}, v_{2} \in \hat{V}$ are contained in a hyperedge $h \in \hat{H}$ whenever they are contained in $h$ in $\Gamma$, and the coefficient $C_{v, h}$ is then taken from $\Gamma$. An induced subhypergraph is also called a motif of $\Gamma$.

Let $\hat{\Gamma}$ be a motif in $\Gamma$. We then have the induced Laplacian

$$
L_{\Gamma, \hat{\Gamma}} f(v)=f(v)+\frac{1}{\operatorname{deg}_{\Gamma} v} \cdot\left(\sum_{h \in \hat{H}} \sum_{v^{\prime} \in \hat{V} \backslash\{v\}} C_{v, h} \cdot C_{v^{\prime}, h} \cdot f\left(v^{\prime}\right)\right)
$$

where $\operatorname{deg}_{\Gamma} v$ denotes the degree of $v$ in $\Gamma$.

Definition 9.5. We say that the motif $\Gamma^{\prime}$ with vertex set $V^{\prime}$ is a duplicated motif if $V^{\prime}$ and $V^{\prime \prime}$ are disconnected, that is, when there is no hyperedge containing elements from both $V^{\prime}$ and $V^{\prime \prime}$.

We say that $\Gamma^{\prime}$ and $\Gamma^{\prime \prime}$ with vertex sets $V^{\prime}$ and $V^{\prime \prime}$ are (anti)twin motives if for every $h \in H$ we have that $v^{\prime} \in h$ iff $v^{\prime \prime}=\tau\left(v^{\prime}\right) \in h$ and $C_{v^{\prime}, h}=C_{v^{\prime \prime}, h}\left(=-C_{v^{\prime \prime}, h}\right)$ for every $v^{\prime} \in V^{\prime}$. 
Lemma 9.6. Let $\Gamma^{\prime}$ be a duplicated motif in $\Gamma$, and let $v_{o} \in V_{o}$ be a neighbor of some $v^{\prime} \in V^{\prime}$. Then $v_{o}$ is also a neighbor of $v^{\prime \prime}=\tau\left(v^{\prime}\right) \in V^{\prime \prime}$.

Proof. Since $v_{0} \in V_{0}$ is fixed by the automorphism $\tau$, and since $\tau$ maps the hyperedge $h$ containing $v_{0}$ and $v^{\prime}$ onto some hyperedge $\tau(h)$ containing $v^{\prime \prime}=\tau\left(v^{\prime}\right)$ and $v_{0}=$ $\tau\left(v_{0}\right)$, the claim follows.

Lemma 9.7. Let $\Gamma^{\prime}$ be a duplicated motif in $\Gamma$. Then we find a basis of eigenfunctions of the Laplacian $L$ of $\Gamma$ of functions $f$ satisfying either

1.

$$
L_{\Gamma, \Gamma^{\prime}} f(v)= \begin{cases}\lambda f(v) & \text { for } \left.v \in V^{\prime} \quad \text { (and also for } v \in V^{\prime \prime}\right) \\ -s(v) f(\tau(v)) & \text { for } v \in V^{\prime \prime} \\ 0 & \text { for } v \in V_{0}\end{cases}
$$

2. or

$$
f(\tau(v))=s(v) f(v) \text { for } v \in V^{\prime} .
$$

The latter eigenfunctions are those of the hypergraph $\Gamma^{\tau}$ obtained as the quotient of $\Gamma$ by $\tau$, that is, the hypergraph with vertex set $V_{0} \cup V^{\prime}$ and all hyperedges induced by $\Gamma$ and the coefficients $C_{v, h}^{\tau}=\sqrt{2} C_{v, h}$.

Proof. If $v_{0}$ in $V_{0}$ and $f\left(v_{0}\right)=0$ and if $f$ is antisymmetric, then also $L f\left(v_{0}\right)=0$, because the contributions from its neighbors $v^{\prime}$ and $v^{\prime \prime}=\tau\left(v^{\prime}\right)$ (see Lemma 9.6) cancel each other in $L f\left(v_{0}\right)$. The result then follows from Lemma 9.3 , because under our assumptions, a neighbor $w$ of $v \in V^{\prime}$ is contained either in $V^{\prime}$ or in $V_{0}$, in which case for an antisymmetric $f, f(w)=0$, and therefore, we can restrict the computation in (20) to the induced Laplacian, that is, consider only the vertices in $V^{\prime}$.

Let us consider some Examples:

1. Let $v^{\prime}$ be a duplicated vertex, that is, $V^{\prime}$ consists only of that single vertex. Then the induced Laplacian $L_{\Gamma, \Gamma^{\prime}} f\left(v^{\prime}\right)=f\left(v^{\prime}\right)$, and therefore, the function $f$ with $f\left(v^{\prime}\right)=1, f\left(v^{\prime \prime}\right)=-1, f(v)=0$ for all other vertices is an eigenfunction with $\lambda=1$. This was shown in [34] and Prop. 8.9.

2. Consider a graph, and let $V^{\prime}$ again consist of a single vertex $v^{\prime}$, but assume that $v^{\prime}$ and $v^{\prime \prime}=\tau\left(v^{\prime}\right)$ are connected by an edge. Then (20) becomes

$$
f\left(v^{\prime}\right)-\frac{1}{\operatorname{deg}_{\Gamma} v^{\prime}} f\left(v^{\prime \prime}\right)=\lambda f\left(v^{\prime}\right)
$$

that is, since $f\left(v^{\prime \prime}\right)=-f\left(v^{\prime}\right)$,

$$
\lambda=1+\frac{1}{\operatorname{deg}_{\Gamma} v^{\prime}} .
$$


3. Let $e=\left(v_{1}^{\prime}, v_{2}^{\prime}\right)$ be a duplicated edge in a graph. Then we can determine two eigenvalues and eigenfunctions of $L$ by solving

$$
\begin{aligned}
f\left(v_{1}^{\prime}\right)-\frac{1}{\operatorname{deg}_{\Gamma} v_{1}^{\prime}} f\left(v_{2}^{\prime}\right)= & \lambda f\left(v_{1}^{\prime}\right) \\
f\left(v_{2}^{\prime}\right)-\frac{1}{\operatorname{deg}_{\Gamma} v_{2}^{\prime}} f\left(v_{1}^{\prime}\right)= & \lambda f\left(v_{2}^{\prime}\right) \\
f(v)=0 & \text { for all other } v .
\end{aligned}
$$

This yields 34

$$
\lambda=1 \pm \frac{1}{\sqrt{\operatorname{deg}_{\Gamma} v_{1}^{\prime} \operatorname{deg}_{\Gamma} v_{2}^{\prime}}} .
$$

Acknowledgments. RM was supported by The Alan Turing Institute under the EPSRC grant EP/N510129/1.

\section{References}

[1] E. Estrada. The structure of complex networks. Oxford Univ. Press, 2012.

[2] F. Battiston, G. Cencetti, I. Iacopini, V. Latora, M. Lucas, A. Patania, J.-G. Young, and G. Petri. Networks beyond pairwise interactions: structure and dynamics. Physics Reports, 2020.

[3] A. Banerjee and J. Jost. Spectral plot properties: Towards a qualitative classification of networks. Networks and Heterogeneous Media, 3(2):395-411, 2008.

[4] J. Jost and R. Mulas. Hypergraph Laplace operators for chemical reaction networks. Advances in Mathematics, 351:870-896, 2019.

[5] F. Chung. Spectral graph theory. American Mathematical Society, 1997.

[6] R. Mulas, C. Kuehn, and J. Jost. Coupled dynamics on hypergraphs: Master stability of steady states and synchronization. Phys. Rev. E, 101:062313, 2020.

[7] R. Mulas. Sharp bounds for the largest eigenvalue of the normalized hypergraph Laplace Operator. Mathematical notes, 2020. To appear.

[8] R. Mulas and D. Zhang. Spectral theory of Laplace Operators on chemical hypergraphs. arXiv:2004.14671.

[9] E. Andreotti and R. Mulas. Spectra of Signless Normalized Laplace Operators for Hypergraphs. arXiv:2005.14484.

[10] J. Jost, R. Mulas, and D. Zhang. p-Laplace Operators for Chemical Hypergraphs. arXiv:2007.00325.

[11] R. Mulas. Spectral classes of hypergraphs. arXiv:2007.04273. 
[12] A. Abiad, R. Mulas, and D. Zhang. Coloring the normalized Laplacian for oriented hypergraphs. arXiv:2008.03269.

[13] R. Mulas, R.J. Sánchez-García, and B.D. MacArthur. Hypergraph Automorphisms. arXiv:2010.01049.

[14] N. Reff and L.J. Rusnak. An oriented hypergraphic approach to algebraic graph theory. Linear Algebra and its Applications, 437:2262-2270, 2012.

[15] L.J. Rusnak. Oriented hypergraphs: Introduction and balance. The Electronic Journal of Combinatorics, 20, 2013.

[16] N. Reff. Spectral properties of oriented hypergraphs. Electronic Journal of Linear Algebra, 27, 2014.

[17] V. Chen, A. Rao, L.J. Rusnak, and A. Yang. A characterization of oriented hypergraphic balance via signed weak walks. Linear Algebra and Its Applications, 485:442-453, 2015.

[18] N. Reff. Intersection graphs of oriented hypergraphs and their matrices. Australasian Journal of Combinatorics, 65(1):108--123, 2016.

[19] E. Robinson, L.J. Rusnak, M. Schmidt, and P. Shroff. Oriented hypergraphic matrix-tree type theorems and bidirected minors via Boolean order ideals. Journal of Algebraic Combinatorics, 2017.

[20] G. Chen, V. Liu, E. Robinson, L.J. Rusnak, and K. Wang. A characterization of oriented hypergraphic Laplacian and adjacency matrix coefficients. Linear Algebra and its Applications, 556:323-341, 2018.

[21] W. Grilliette and L.J. Rusnak. Incidence hypergraphs: The categorical inconsistency of set-systems and a characterization of quiver exponentials. arXiv:1805.07670.

[22] L. Duttweiler and N. Reff. Spectra of cycle and path families of oriented hypergraphs. Linear Algebra and its Applications, 578:251-271, 2019.

[23] O. Kitouni and N. Reff. Lower bounds for the Laplacian spectral radius of an oriented hypergraph. Australasian Journal of Combinatorics, 74(3):408 -422, 2019.

[24] W. Grilliette, J. Reynes, and L.J. Rusnak. Incidence hypergraphs: Injectivity, uniformity, and matrix-tree theorems. arXiv:1910.02305, 2019.

[25] L.J. Rusnak, S. Li, B. Xu, E. Yan, and S. Zhu. Oriented Hypergraphs: Balanceability. arXiv:2005.07722.

[26] W. Grilliette and L.J. Rusnak. Incidence Hypergraphs: Box Products \& the Laplacian. arXiv:2007.01842.

[27] A. Banerjee and S. Parui. On synchronization in coupled dynamical systems on hypergraphs. arXiv:2008.00469, 2020. 
[28] J. Jost. Riemannian geometry and geometric analysis. Springer, 7th edition, 2017.

[29] S. Klamt and J. Stelling. Two approaches for metabolic pathway analysis? Trends in Biotechnology, 21, 2003.

[30] R. A. Horn and C. R. Johnson. Matrix Analysis. Cambridge University Press, second edition, 2013.

[31] B.D. MacArthur, R.J. Sánchez-García, and J.W. Anderson. Symmetry in complex networks. Discrete Applied Mathematics, 156(18):3525-3531, 2008.

[32] B.D. MacArthur and R.J. Sánchez-García. Spectral characteristics of network redundancy. Physical Review E, 80(2), 2009.

[33] R.J. Sánchez-García. Exploiting symmetry in network analysis. Commun. Phys., 3(87), 2020.

[34] A. Banerjee and J. Jost. On the spectrum of the normalized graph Laplacian. Linear Algebra and its Applications, 428(11-12):3015-22, 2008. 